\title{
Rodríguez-Blanco sobre el carácter inestable del punto de vista interno
}

\author{
Rodriguez-Blanco on the Instability of the \\ Internal Point of View
}

Ezequiel Monti*

\begin{abstract}
Resumen: En el artículo publicado en el presente volumen, Verónica Rodríguez-Blanco argumenta que el punto de vista interno hartiano es inestable en la medida que, al centrarse en los estados mentales del agente, solo puede conducirnos a comprender el sentido o propósito de las acciones de un modo accidental. El núcleo del argumento es que el punto de vista interno presupone un enfoque inward-looking de la acción y que, para comprender adecuadamente el sentido o propósito de las acciones, es necesario adoptar, en cambio, un enfoque outward-looking. En este artículo, sostengo que los argumentos de Rodríguez-Blanco en contra del enfoque inward-looking de la acción son insatisfactorios. Argumento, además, que las críticas que dirige a la teoría del punto de vista jurídico de Raz están igualmente desencaminadas, y que su propuesta positiva relativa al rol de las reglas jurídicas en el razonamiento práctico tiene serios defectos.
\end{abstract}

Palabras clave: punto de vista interno, punto de vista jurídico, normatividad del derecho.

* Profesor Asistente, Universidad Torcuato di Tella (UTDT), Buenos Aires, Argentina. Correo electrónico: emonti@utdt.edu 


\begin{abstract}
In her contribution to the present volume, Verónica Rodríguez-Blanco argues that Hart's internal point of view is instable insofar as understanding the mental states of an agent can only lead us to understand the point of her action by accident or sheer coincidence. In a nutshell, the argument is that the internal point of view presupposes an inward-looking approach to action and that, to adequately understand the point of actions, it is necessary to adopt an outward-looking approach instead. In this paper, I argue that Rodríguez-Blanco's arguments against the inward-looking approach to action are unsuccessful. Furthermore, I argue that her criticisms of Raz's legal point of view are equally misguided, and that her own positive account of the role of legal rules in practical reasoning suffers from serious defects.
\end{abstract}

Keywords: Internal point of view, legal point of view, normativity of Law.

\title{
1. Introducción: la crítica de Finnis a Hart y el argumento de Rodríguez-Blanco
}

En Natural Law and Natural Rights, John Finnis argumenta que el enfoque metodológico de H. L. A. Hart debe ser rechazado. Según Finnis, la idea básica del enfoque hartiano es que, a efectos de evaluar qué rasgos del derecho son teóricamente importantes o significativos, debe darse prioridad a las preocupaciones y evaluaciones de quienes adoptan el punto de vista interno. Brevemente, Finnis argumenta que dicho enfoque metodológico es "inestable e insatisfactorio" (Finnis, 1980, p. 13). El argumento, tal como yo lo entiendo, es el siguiente.

Hart distingue, correctamente, entre aquellas personas que adoptan el punto de vista externo, esto es, que consideran que el derecho es prácticamente relevante únicamente en la medida en que existe un riesgo de ser sancionado si uno no actúa como requiere, y aquellas que, en cambio, adoptan el punto de vista interno, esto es, que consideran que las reglas jurídicas constituyen en sí mismas razones para actuar y que consideran que es apropiado exigir a los demás que actúen como requieren así como reprochar a quienes no lo hacen sin excusa ni justificación. Y, también correctamente, Hart otorga prioridad explicatoria al punto de vista interno 
sobre el punto de vista externo (Finnis, 1980, p. 12). Sin embargo, exactamente las mismas razones que llevan a trazar esta distinción entre el punto de vista externo y el punto de vista interno, dándose prioridad a este último, justifican igualmente trazar distinciones ulteriores entre diversas instancias del punto de vista interno mismo, dándose prioridad a una de ellas. Así, por ejemplo, corresponde distinguir entre quienes aceptan el derecho por razones puramente autointeresadas y quienes lo aceptan porque consideran que tiene autoridad moral (i. e. que existe una obligación moral de actuar como requiere) y juzgan que su establecimiento y mantenimiento es una exigencia de justicia. Y está claro, dice Finnis, que el primero constituye una instancia marginal o periférica del punto de vista interno mientras que el segundo es una instancia paradigmática o central, por lo que debe otorgarse prioridad explicatoria a esta última (Finnis, 1980, pp. 14-15). Por ello, concluye Finnis, el enfoque de Hart es inestable. Una vez que se rechaza como periférico o marginal al punto de vista externo, ¿̨por qué no habría de rechazarse como igualmente marginales a variantes del punto de vista interno como la mencionada, dándose prioridad al punto de vista de quienes consideran que el derecho tiene autoridad moral? El enfoque es, además, insatisfactorio en la medida que implica negar relevancia teórica a distinciones que cualquier participante reconocería como prácticamente relevantes (por ejemplo: tanto quienes aceptan el derecho por razones puramente autointeresadas como quienes lo aceptan en virtud de su autoridad moral entienden que es una cuestión de suma importancia práctica cuál de estas dos actitudes prácticas se adopta) (Finnis, 1980, p. 13).

En el artículo publicado en el presente volumen, el objetivo declarado de Verónica Rodríguez-Blanco (2021) es explorar esta línea argumentativa en mayor profundidad. Así, sostiene que la crítica de Finnis puede ser reconducida a una más fundamental. Según esta crítica más fundamental, el punto de vista interno hartiano no puede ser utilizado para comprender el propósito (point) de las acciones humanas en el contexto jurídico. O, en otros términos, la teoría de Hart está edificada sobre una teoría de la acción falsa, inadecuada o insuficiente. Sería en virtud de este defecto más fundamental que la propuesta metodológica de Hart deviene necesariamente inestable e insatisfactoria. El argumento de Rodríguez-Blanco puede ser reconstruido del siguiente modo: 
(1) Una teoría del derecho adecuada debe ser capaz de comprender las acciones en el contexto jurídico.

(2) Comprender una acción implica comprender su propósito o sentido (i. e. su "point").

(3) Por lo tanto, una teoría del derecho adecuada debe ser capaz de comprender el propósito o sentido de las acciones en el contexto jurídico [de (1) y (2)].

(4) El punto de vista interno hartiano se refiere únicamente a los estados mentales del agente que guía su conducta en virtud de reglas (sea la regla de reconocimiento o cualquier regla social o jurídica), en particular, a la actitud crítica reflexiva que Hart llama "aceptación".

(5) Comprender los estados mentales del agente al realizar una acción no es equivalente a comprender su propósito o sentido. Comprender los estados mentales del agente puede, accidentalmente, llevar a comprender su propósito o sentido. Pero, en ese caso, se trata solo de una coincidencia.

(6) El punto de vista interno hartiano solo puede llevar a comprender el propósito o sentido de las acciones por accidente. Es, en este sentido, inestable [de (4) y (5)].

(7) Por lo tanto, la teoría del derecho de Hart es inadecuada y debe ser rechazada [de (3) y (6)].

La premisa crucial es (5). Rodríguez-Blanco justifica esta premisa argumentando en contra del enfoque "inward-looking" de la acción característico de las teorías que, como la de Donald Davidson, entienden a las acciones intencionales como acciones que son causadas por determinados estados mentales del agente (por ejemplo, por sus creencias y deseos o actitudes favorables) (Davidson, 2001, pp. 12-25). La idea es que el punto de vista interno hartiano presupone un enfoque de este tipo; y que, por lo tanto, padece de los mismos defectos. Rodríguez-Blanco defiende, en cambio, un enfoque "outward-looking" de la acción, característico de teorías que, como la de Elizabeth Anscombe, sostienen que alguien actúa intencionalmente cuando lo hace por una razón, esto es, un rasgo o propiedad de la acción en virtud del cual (considera que) es bueno o valioso realizarla (Anscombe, 1963). 
En lo que sigue, procederé del siguiente modo. En primer lugar, realizaré dos aclaraciones preliminares (sección 2). En este sentido, $i$ ) introduciré algunas razones para dudar que la reconstrucción que realiza Finnis de la metodología de Hart sea correcta; y ii) realizaré algunas observaciones acerca de la conexión entre el argumento de Finnis y el de Rodríguez-Blanco. En segundo lugar, reconstruiré los argumentos centrales de Rodríguez-Blanco en contra del enfoque inward-looking de la acción y argumentaré que deben ser rechazados (sección 3). En tercer lugar, criticaré brevemente el argumento de Rodríguez-Blanco en contra de la teoría del punto de vista jurídico de Joseph Raz, cuya noción central es la de enunciados normativos no comprometidos (detached normative statements), y a la que Rodríguez-Blanco considera como una alternativa tanto a su propio enfoque metodológico, centrado en el punto de vista deliberativo, como al de Hart, centrado en el punto de vista interno (sección 4). Por último, cerraré realizando algunas observaciones críticas acerca del modo en que Rodríguez-Blanco reconstruye el rol de las reglas jurídicas en el razonamiento práctico (sección 5 ).

\section{Algunas observaciones/advertencias preliminares}

Déjenme comenzar por una breve aclaración con relación al modo en que Finnis reconstruye el enfoque metodológico de Hart. Finnis sostiene que Hart otorga prioridad teórica al punto de vista interno (Finnis, 1980, p. 12). Esto implica, dice Finnis, que las preocupaciones y evaluaciones de quienes adoptan el punto de vista interno tienen prioridad al momento de identificar qué rasgos de la práctica jurídica son teóricamente importantes o significativos. Sin embargo, no creo que esta reconstrucción de Hart sea correcta. Es cierto que Hart rechaza el análisis predictivo de las obligaciones porque no reconstruye adecuadamente el punto de vista de interno. Sin embargo, Hart también sostiene enfáticamente que una teoría adecuada del derecho debe hacer justicia a ambos puntos de vista (Hart, 2012, p. 91). Una teoría que no permita reconstruir adecuadamente el punto de vista de, digamos, el anarquista que cree que el derecho no es en absoluto vinculante sería igualmente inadecuado. Así, para el teórico hartiano tanto el punto de vista del 
aceptante como el del alienado son relevantes a efectos de determinar qué rasgos de la práctica jurídica son teóricamente importantes o significativos. Las preocupaciones y evaluaciones de ambos deben ayudarnos a identificar los rasgos o aspectos importantes y significativos de la práctica.

Es cierto que Hart otorga cierta prioridad al punto de vista interno. En este sentido, Hart sostiene que no es posible que exista derecho a menos que algunas personas (los funcionarios o los jueces) adopten el punto de vista interno con respecto a algunas reglas (la regla de reconocimiento) $y$, en cambio, es perfectamente posible que exista derecho sin que nadie adopte el punto de vista externo (Hart, 2012, p. 116). Pero esta es una prioridad ontológica, no metodológica.

La segunda aclaración preliminar es la siguiente. Rodríguez-Blanco sostiene que su crítica al punto de vista interno hartiano es un desarrollo, a un nivel más básico, de la crítica de Finnis. Sin embargo, debo confesar que soy incapaz de ver la conexión entre ambos argumentos. Como vimos, el argumento de Finnis es que una vez que uno distingue entre el punto de vista externo y el punto de vista interno debería, por las mismas razones, distinguir entre diversas instancias del punto de vista interno. La metodología de Hart sería inestable precisamente porque realiza la primera distinción, pero se rehúsa a trazar distinciones ulteriores entre diversas instancias del punto de vista interno. La crítica de Rodríguez-Blanco, a primera vista, tiene poco que ver con esta. El argumento de Rodríguez-Blanco es que, en la medida en que se centra exclusivamente en los estados mentales de los agentes, el punto de vista hartiano carece de los elementos necesarios para comprender adecuadamente las acciones de los agentes en el contexto jurídico. Rodríguez-Blanco también afirma que la teoría de Hart es, por ello, inestable. Pero la inestabilidad a la que se alude es, aparentemente, de un carácter completamente diferente. El punto de vista interno hartiano sería inestable porque solo accidentalmente puede conducir a comprender el propósito o sentido de las acciones en el contexto jurídico. Esto, a primera vista, no tiene mucho que ver con la inestabilidad que denuncia Finnis. En todo caso, sería bueno que Rodríguez-Blanco clarificara explícitamente este punto. 


\section{Los argumentos de Rodríguez-Blanco contra el enfoque de la acción que subyace al punto de vista interno hartiano}

Como mencioné en la introducción, la tesis central de Rodríguez-Blanco es que el enfoque metodológico de Hart, centrado en la noción de punto de vista interno, es inestable en la medida en que comprender los estados mentales de los agentes solo puede llevarnos a comprender el sentido o propósito de sus acciones de un modo accidental. El núcleo del argumento es que el punto de vista interno hartiano presupone un enfoque inward-looking de la acción y que, para comprender adecuadamente el sentido o propósito de las acciones, es necesario adoptar, en cambio, un enfoque outward-looking. Rodríguez-Blanco introduce tres argumentos centrales que intentan demostrar la falsedad, inadecuación o limitaciones del enfoque inward-looking de la acción, a saber, el argumento de las cadenas causales desviadas (sección 3.2), el argumento de la memoria (sección 3.3) y el argumento de la transparencia (secciones 3.4. y 3.5). Aquí argumentaré que ninguno de estos argumentos es exitoso.

\subsection{Introducción al enfoque inward-looking}

La idea básica de los enfoques inward-looking es que las acciones intencionales deben ser analizadas en términos de los estados mentales que las explican. De acuerdo a este enfoque, una acción es intencional precisamente porque es explicada de cierta manera por estados mentales de cierto tipo. La teoría causal es probablemente la teoría más famosa en esta línea. Sintéticamente, de acuerdo a la teoría causal de la acción:

Teoría Causal de la Acción: A pa intencionalmente si y solo si:

(i) A cree que realizando $\varphi$ realizará $\psi$;

(ii) A tiene una actitud favorable hacia $\psi$; y

(iii) Los estados mentales (i) y (ii) causan que $\mathrm{A} \varphi \mathrm{e}$. 


\subsection{El argumento de las cadenas causales desviadas}

Uno de los argumentos centrales de Rodríguez-Blanco en contra de la teoría causal de la acción es el de las cadenas causales desviadas. La idea es que hay casos en que las tres condiciones (i)-(iii) están satisfechas, pero en los que, intuitivamente, no diríamos que la persona en cuestión actuó intencionalmente. Considérese el siguiente ejemplo: ${ }^{1}$

El Montañista: Un montañista quiere dejar caer a su compañero, a quien sostiene con una soga, porque de ese modo se librará del peso correspondiente (de lo contrario, existe un alto riesgo de que ambos terminen cayendo). Cree que, si abre ligeramente su mano, aflojando de ese modo su agarre de la soga, dejará caer a su compañero. Este conjunto de creencias y deseos lo pone nervioso, lo que causa que abra ligeramente la mano, dejando caer a su compañero.

La creencia y el deseo del montañista causaron que abra la mano y suelte a su compañero, pero, intuitivamente, nadie diría que el montañista abrió la mano o dejó caer a su compañero intencionalmente. El no decidió abrir la mano y dejarlo caer: fueron sus nervios.

Los defensores de la teoría causal de la acción reconocen que este es un problema serio.

Una solución sería sustituir (iii) por:

(iii) Los estados mentales (i) y (ii) causan, de un modo apropiado, que A $\varphi$ e.

La idea es, entonces, que, aunque los estados mentales del montañista causan que abra ligeramente la mano, no lo hacen del modo apropiado: se trata de una cadena causal desviada. Pero, por supuesto, esta respuesta es incompleta. Debería proporcionarse un conjunto de criterios que nos permitan distinguir entre cadenas causales apropiadas y desviadas de un modo que no sea ad hoc (esto es, no debe ser el caso que los criterios estén moti-

1 El ejemplo es de Davidson. Véase Davidson (2001, p. 73). Rodríguez-Blanco proporciona, además, un ejemplo análogo en el contexto jurídico (Rodríguez-Blanco, 2021, sección 3). 
vados solo a efectos de evitar los contraejemplos). Rodríguez-Blanco parece suponer, empero, que este desafío no puede responderse adecuadamente.

Hasta aquí, entonces, el argumento de Rodríguez-Blanco. Tengo mis dudas acerca de que la teoría causal de la acción no tenga nada que ofrecer para distinguir entre cadenas causales desviadas y apropiadas. Pero aquí me centraré en otra cosa. Ciertamente, Hart adopta un enfoque inward-looking de la acción en la medida que reconstruye el punto de vista interno en términos de los estados mentales (aceptación) del agente. Pero, hasta donde yo sé, en ningún momento se compromete con una teoría causal de la acción. Es perfectamente compatible con la teoría hartiana la adopción de un enfoque inward-looking no causal. Considérese, por ejemplo:

Teoría Inward-Looking No-Causal de la Acción: A pa intencionalmente si y solo si:

(i) A cree que se obtiene un hecho $\mathrm{H}$;

(ii) A cree que $\mathrm{H}$ cuenta en favor de realizar $\varphi$ (o: A tiene una actitud favorable hacia $\varphi$ en virtud de $\mathrm{H})$; $\mathrm{y}$

(iii) Los estados mentales (i) y (ii) explican no causalmente que A pe.

Esta teoría es inward-looking en la medida en que se centra en estados mentales. Pero no es causal y, por ende, no está expuesta al argumento de las cadenas causales desviadas. El montañista no actúa intencionalmente porque, aunque los estados mentales explican por qué el montañista abrió la mano, lo hacen de un modo causal antes que no-causal. Así, la crítica de Rodríguez-Blanco solo se aplica a la teoría causal de la acción, pero no a otras teorías inward-looking que son igualmente compatibles con el enfoque hartiano.

\subsection{El argumento de la memoria}

Rodríguez-Blanco (2021, sección 2) presenta de un modo sucinto un segundo argumento en contra del enfoque inward-looking. Este es un argumento intrigante basado en algunos famosos (e igualmente intrigantes) pasajes de Ludwig Wittgenstein (1967, $₫ 645$ y ss.). Hay aquí, creo, tres objeciones relacionadas. La idea es que, si tener una intención es tener un estado mental, entonces: 
i. Uno debería ser capaz de recordar ese estado mental y reflexionar sobre él; pero no somos capaces de recordar un estado mental de "intentar" o "tener la intención" que sea independiente de nuestros otros pensamientos, emociones, etc., al realizar la acción.

ii. Las intenciones tendrían, como los estados mentales, un comienzo y un fin determinados; pero esto es implausible. Por ejemplo: ¿cuándo, precisamente, termina mi intención de escribir este comentario? ¿Cuándo empiezo a escribirlo? ¿Cuándo escribo la última letra? ¿Cuándo lo imprimo?

iii. Muchas veces interrumpimos una acción intencional y luego la continuamos. Si las intenciones fueran estados mentales, parece que para retomar una acción intencional que había iniciado antes debería recordar o retomar el estado mental en el que estaba inicialmente. Pero esto es implausible: "una intención puede ser llevada a cabo en cualquier momento, pero un estado mental puede olvidarse o desaparecer por completo" (Rodríguez-Blanco, 2014, p. 44).

Estas observaciones son, creo, aproximadamente correctas e iluminadoras. Sin embargo, el enfoque inward-looking no está necesariamente comprometido con la tesis de que las intenciones son estados mentales distintos e independientes. Según el enfoque inward-looking, alguien actúa intencionalmente cuando sus acciones son explicadas de cierta manera por sus estados mentales. No necesita postular que hay un estado mental que se corresponda con la "intención". Consideremos los dos enfoques inward-looking que examiné hasta ahora. No afirman que hay un estado mental de "tener la intención" además o por encima de las creencias y actitudes favorables del agente. Tampoco dice que tener esos estados mentales es suficiente para tener la intención. Uno actúa intencionalmente solo cuando esos estados mentales explican nuestra acción de una determinada manera. Por lo tanto, nuevamente, aunque estas críticas sean adecuadas, Hart no está necesariamente comprometido con una teoría de la acción que sea vulnerable a ellas. 


\subsection{El argumento de la transparencia}

Un tercer argumento de Rodríguez-Blanco (2021, sección 3) en contra del enfoque inward-looking es el siguiente. Cuando un agente delibera acerca de qué hacer no examina sus propios estados mentales, no examina sus creencias y actitudes, sino que examina el mundo, examina qué hechos se obtienen y qué cuenta a favor o en contra de tal o cual acción. Lo mismo sucede cuando le preguntamos a alguien por qué está haciendo lo que está haciendo: no responderá apelando a sus estados mentales sino a los hechos que (a su juicio) justifican actuar como está actuando. Supongamos que Juan entra a una habitación, ve a una serpiente venenosa, y sale corriendo. Nosotros lo vemos salir corriendo de la habitación y le preguntamos por qué corre. Su respuesta no será en términos de sus creencias y actitudes. Nos dirá que está corriendo porque hay una serpiente venenosa en la habitación (no que está corriendo porque cree que hay una serpiente venenosa y tiene una actitud desfavorable hacia las serpientes venenosas o algo de ese estilo). De hecho, tenemos un conocimiento no observacional (no basado en evidencia alguna) de que actuamos intencionalmente y de las razones por las que actuamos. No necesitamos considerar nuestros estados mentales para saber si estamos actuando intencionalmente o las razones por las que estamos actuando. Basta con que consideremos los hechos externos que son razones para actuar como estamos actuando. En este sentido, mi creencia de que estoy actuando intencionalmente por determinadas razones es transparente.

La idea de transparencia relevante aquí puede explicarse del siguiente modo. Consideremos el sentido en el que las creencias son transparentes. La idea es que, desde la perspectiva de primera persona, tratamos de manera idéntica a las preguntas “¿Creo que está lloviendo?” y “Está lloviendo?". Para determinar si creo que está lloviendo, miro a través de la ventana, no considero mis estados mentales. La pregunta teórica acerca de mis estados mentales es deferida a la pregunta deliberativa acerca de lo que debo o he de creer. La idea es que las razones para la acción son transparentes en el mismo sentido: desde la perspectiva de primera persona, tratamos de manera idéntica a las preguntas “¿Por qué razones estoy actuando?” y 
“Qué razones cuentan a favor de hacer lo que estoy haciendo?”. La pregunta teórica acerca de las razones que me motivan a actuar es deferida a la pregunta teórica acerca de lo que debo o he de hacer.

De nuevo, creo que todo esto es aproximadamente correcto. La pregunta es qué se sigue exactamente respecto al enfoque inward-looking. En Law and Authority Under the Guise of the Good, Rodríguez-Blanco argumenta que la explicación naif de la acción, esto es, la explicación desde el punto de vista deliberativo en términos de las características en virtud de las cuales es bueno o valioso realizar la acción, tiene prioridad sobre la explicación teórica de la acción en términos de los estados mentales del agente. $\mathrm{O}$, en términos ligeramente distintos, sostiene que la primera explicación es parasitaria o depende de la primera (Rodríguez-Blanco, 2014, caps. 5 y 6$)$.

En un sentido, esto es correcto. Los agentes actúan en virtud de hechos del mundo que, a su juicio, cuentan a favor de realizar las acciones que realizan. La explicación teórica intenta describir este fenómeno desde un punto de vista de tercera persona. Y, para ello, debe apelar a determinada reconstrucción de cómo se ve el mundo desde la perspectiva deliberativa del agente cuya acción se está explicando.

Pero nada de esto implica que el enfoque inward-looking sea falso, incorrecto o inadecuado. Simplemente muestra que las dos explicaciones, desde el punto de vista deliberativo en términos de las características en virtud de las cuales es bueno o valioso realizar la acción, y desde el punto de vista teórico en términos de estados mentales, no son explicaciones alternativas y excluyentes. Son, por el contrario, complementarias.

Más aún, que las explicaciones teóricas en términos de estados mentales dependan de este modo de las explicaciones desde el punto vista deliberativo no implica que las primeras sean innecesarias. Son, por el contrario, un complemento necesario de las segundas. Ello se pone claramente de manifiesto cuando los agentes actúan por razones que, en realidad, no tenían. Esto es, cuando actúan en virtud de que creen que existe un hecho que cuenta a favor de realizar una acción, pero, en realidad, dicho hecho no existe o no cuenta efectivamente a favor de la acción. Supongamos que la serpiente que Juan creyó ver en la habitación era, en realidad, un juguete. Cuando Juan nos dice que está corriendo porque hay una serpiente vene- 
nosa en la habitación, nosotros, que conocemos que la serpiente era de juguete, comenzamos a reírnos. Cuando Juan cuenta la anécdota en la cena no dirá que salió corriendo porque había una serpiente. Esa explicación ya no está disponible porque, de hecho, no había ninguna serpiente. Para que una explicación sea adecuada sus premisas deben ser verdaderas. Juan dirá, en cambio, que salió corriendo porque creía que había una serpiente, esto es, apelará a sus estados mentales, antes que al mundo. No tendrá, en efecto, otra explicación disponible.

Ahora supongamos que queremos explicar por qué Juan salió corriendo sin comprometernos con la existencia de la serpiente. Entonces, nuevamente, no nos queda otra opción que explicar su acción en virtud de sus creencias y actitudes antes que en virtud de como él mismo hubiera explicado su acción en el momento en que estaba realizándola, esto es, en términos de serpientes en lugar de creencias acerca de serpientes. Esto podría explicar por qué Hart, cuya pretensión consistía en construir una teoría del derecho que haga justicia tanto al punto de vista externo como al interno, se limita a explicar las acciones en el contexto jurídico en términos de estados mentales.

\subsection{Transparencia y privilegio epistémico del punto de vista deliberativo}

¿Pero no hay un costo a pagar por esta neutralidad? Rodríguez-Blanco sugiere que sí. Así, sostiene que las explicaciones teóricas en términos de estados mentales son, en algún sentido, menos ricas o deficientes desde el punto de vista epistémico. Son varias las consideraciones que Rodríguez-Blanco realiza en este sentido. Déjenme centrarme en dos que están relacionadas:

a. Primero, Rodríguez-Blanco sostiene que hay límites a la teorización del punto de vista deliberativo, esto es, que hay un aspecto interno de la acción guiada por razones que no puede ser adecuadamente capturado por las explicaciones en términos de estados mentales. 
b. Segundo, Rodríguez-Blanco sugiere que la descripción que realiza el propio agente acerca de las razones en virtud de la cuales actúa tiene una autoridad epistémica especial y privilegiada (precisamente porque las razones son transparentes desde el punto de vista deliberativo).

Comencemos por (a). Las consideraciones que introduce Rodríguez-Blanco para sustentar esta tesis son extremadamente débiles. De hecho, entiendo que la tesis exactamente opuesta es correcta:

Tesis de la traducción: Para toda acción intencional, es posible "traducir" su explicación desde el punto de vista deliberativo en términos de las características que hacen que sea valioso o bueno realizarla, a una explicación en términos de los estados mentales del agente.

La idea es básicamente la siguiente. Consideremos una acción cualquiera, por ejemplo, el pago del alquiler de Juan. Si le preguntamos a Juan por qué está pagando el alquiler, responderá que lo paga porque había prometido que lo haría y es bueno cumplir con las promesas. Ahora podemos traducir esta explicación en términos de las características que hacen valioso que Juan pague el alquiler a una explicación en términos de los estados mentales de Juan. Simplemente decimos que Juan pagó el alquiler porque creía que lo había prometido y porque creía que es bueno cumplir con las promesas. Esta segunda explicación, es cierto, depende de la primera. Pero no parece que haya ninguna pérdida en la traducción. ¿ O sí? Si no la hay en este caso, ¿hay alguna otra acción cuya explicación deliberativa no pueda ser traducida a una explicación teórica sin pérdida? Eso es, creo, lo que Rodríguez-Blanco debería mostrar.

Pasemos, ahora, a (b). Rodríguez-Blanco sugiere que la descripción del propio agente de las razones por las que actúa es epistémicamente privilegiada. Esto es lo que subyace a la why-question methodology. De hecho, a veces sostiene (siguiendo algún pasaje de Anscombe) que el problema es, simplemente, controlar que la descripción del agente sea sincera (Rodríguez-Blanco, 2021, sección 2). Esto sugiere, a su vez, que el agente es infa- 
lible acerca de las razones por las que actúa. Se trata, creo, de un error. En particular, es un error inferir de:

(1) Los agentes tienen un conocimiento no observacional o no basado en evidencia de las razones por las que actúan (esto es, las razones por las que actúan les son transparentes a los agentes).

que:

(2) Por lo tanto, los agentes tienen conocimiento infalible o un acceso epistémicamente privilegiado a las razones por las que actúan.

Es posible que Juan crea sinceramente que pagó el alquiler porque lo había prometido y es bueno en sí mismo cumplir con las promesas pero que, en rigor, lo que efectivamente explica (desde el punto de vista deliberativo) el hecho de que haya pagado el alquiler haya sido que, de otro modo, la persona a quien le prometió se habría enojado y habría dejado de confiar en él. Juan forma la creencia de que paga el alquiler porque lo prometió de modo no observacional: simplemente examina qué cuenta a favor de qué (en este caso, el hecho de haber prometido pagar el alquiler cuenta a favor de pagarlo). Pero ello no implica que no pueda estar equivocado. Aunque sinceramente crea que cumplir con las promesas es intrínsecamente valioso, y aunque sinceramente crea que esa es la razón por la que paga el alquiler, es posible que lo que efectivamente está guiando su conducta sea otra cosa. Este es, asumo, un fenómeno psicológico familiar. Muchas veces repasamos lo que hicimos y nos damos cuenta que lo que efectivamente guiaba nuestras acciones no era lo que nosotros creíamos que nos guiaba al momento de realizarlas. En este sentido, debemos distinguir entre las razones que motivan al agente, esto es, las razones que el cree que guían su acción al momento de realizarlas, de las razones que efectivamente explican su accionar, las que efectivamente lo guían. Por supuesto, el agente debe estar aware de las razones que efectivamente lo guían (i.e. debe estar aware de que la persona se enojará) y debe creer que efectivamente cuentan a favor de hacer lo que hace. Por ello, Anscombe y Rodríguez-Blanco tienen razón al sostener que si alguien contesta que no estaba aware de que estaba 
haciendo algo, entonces, no estaba haciéndolo intencionalmente. Pero no es necesario que, además, el agente esté aware de que esas son las razones que efectivamente lo guían (i.e. de que actúa porque la persona se enojará).

\subsection{Algunas observaciones adicionales sobre los supuestos defectos epistémicos del punto de vista interno hartiano}

Rodríguez-Blanco realiza dos observaciones adicionales sobre los defectos epistémicos del punto de vista interno hartiano que, creo, vale la pena comentar brevemente.

En primer lugar, afirma que, de acuerdo a Hart, la acción (o el sentido o propósito de la acción) es capturada por un observador externo a través del siguiente mecanismo. Se observa que el agente sigue un patrón de conducta de crítica a las desviaciones de la regla y, de ello, se infiere que acepta dicha regla como un estándar de conducta. Esto es, por supuesto, un error. Alguien puede criticar las desviaciones de la regla por una multitud de razones que no tienen nada que ver con su aceptación de la regla. Pero no creo que esa sea una reconstrucción adecuada de la posición de Hart. Hart explícitamente sostiene que no basta con que alguien exija a otros que actúen conforme a la regla y que los reproche si no lo hacen para considerar que acepta la regla en el sentido relevante. Es necesario que considere que el hecho de que la regla requiere una acción es una razón para realizarla y que las demandas y reproches referidos son apropiados o legítimos. ${ }^{2} \mathrm{Y}$ en

2 Así, por ejemplo, al explicar el aspecto interno de las reglas, Hart sostiene que los jugadores de ajedrez: "tienen una actitud crítica reflexiva hacia este patrón de conducta [de mover a la reina de determinada manera]: lo consideran como un estándar para todos los jugadores. No solo cada uno mueve la reina de determinada manera, sino que también tiene una opinión formada acerca de la corrección de que todos muevan la reina de esa manera. Esas opiniones se manifiestan en la crítica a otros y en las demandas de conformidad dirigidas a otros [...] y en el reconocimiento de la legitimidad de esas críticas y demandas cuando son realizadas por otros" (Hart, 2012, p. 57).

Este pasaje, y otros similares que aparecen a lo largo del libro, dejan en claro que una cosa es la actitud de aceptación (considerar que la regla es un estándar de conducta para el grupo) y otra cosa es la manifestación externa de esa actitud en las demandas y críticas a otros, así como en el reconocimiento público de la legitimidad de esas demandas y críticas. 
ningún momento afirma que la única evidencia relevante para determinar si esta es efectivamente la actitud que tiene una persona hacia la regla sea su patrón de crítica a la conducta de los demás. Hay una multitud de elementos de prueba que deberían considerarse. ${ }^{3}$

En segundo lugar, Rodríguez-Blanco sostiene que, de acuerdo a la metodología de Hart, no podemos distinguir entre sistemas jurídicos malvados y benevolentes o que dicha distinción es irrelevante. Pero esto es simplemente falso y no entiendo por qué Rodríguez-Blanco afirma tal cosa. ¡Por supuesto que la metodología de Hart permite distinguir entre sistemas jurídicos buenos y malos! ¡Y por supuesto que la distinción importa!

3 Así, por ejemplo, Hart sostiene que: "La evidencia de que tales acciones [en las que las personas hacen lo que la regla requiere sin antes pensar en ella] son genuinas aplicaciones de la regla es el contexto en el que ocurren [...] El más importante de estos factores contextuales [...] es que si nuestra conducta fuera desafiada estaríamos dispuestos a justificarla invocando la regla; y el carácter genuino de nuestra aceptación de la regla puede manifestarse no solo en nuestra reconocimiento de ella y en nuestra conformidad con ella (tanto pasada como subsiguiente), sino también en nuestra crítica a las desviaciones de ella (tanto propias como ajenas). En base a evidencia como esa $u$ otra similar, podemos concluir que si [...] nos hubieran preguntado qué era correcto hacer y por qué, habríamos, de ser honestos, invocado la regla en respuesta" (Hart, 2012, p. 140).

Nótese que Hart identifica aquí otros elementos de prueba además de la existencia de un patrón de conducta de crítica a las desviaciones de la regla (por ejemplo, la disposición a invocar la regla en caso de desafío) y que constantemente aclara que los elementos de prueba a los que se refiere no son exhaustivos ("el más importante", "evidencia como esa u otra similar") ni necesariamente concluyente ("podemos"). Asimismo, Hart explícitamente reconoce que es perfectamente posible que la evidencia relevante sugiera que alguien acepta la regla, aunque no lo haga realmente. Así, inmediatamente aclara que: "Esto no quiere decir que la simulación no sea posible y a veces exitosa. Los tests para determinar si alguien meramente simuló ex post facto haber actuado en base a la regla son, como todos los tests empíricos, inherentemente falibles" (Hart, 2012, p. 140). Estos pasajes revelan, creo, que la posición de Hart es que la determinación de si alguien acepta realmente una regla o meramente simula hacerlo es una cuestión epistemológica compleja, en la que deben sopesarse elementos de prueba de diversa índole, sin que ninguno de ellos sea necesariamente concluyente, y en la que, por más cuidadosos que seamos, siempre es posible que arribemos a conclusiones equivocadas. Esta es, creo, una imagen muy distinta de la que sugiere Rodríguez-Blanco. 


\section{Sobre la crítica de Rodríguez-Blanco al punto de vista normativo no comprometido de Raz}

Rodríguez-Blanco considera brevemente el punto de vista jurídico de Raz como una alternativa metodológica tanto al punto de vista deliberativo (porque, a diferencia de este, no implica compromisos normativos) como al punto de vista teórico hartiano (porque, a diferencia de este, no se refiere a los estados mentales de los agentes, sino que es normativo, esto es, se refiere a lo que los agentes tienen razón para hacer).

La objeción de Rodríguez-Blanco a esta alternativa está fundada, creo, en un error de interpretación. Rodríguez-Blanco comienza analizando el siguiente famoso ejemplo de Raz:

Mi amiga vegetariana: Ezequiel es omnívoro. No cree que haya ninguna razón para abstenerse de comer carne. Sin embargo, le dice a su amiga vegetariana, Verónica, que no debería comer ese sándwich porque tiene carne.

Este es un fenómeno cotidiano y familiar. Es, empero, difícil de explicar. ¿Cómo puede ser que Ezequiel le diga a Verónica que debería abstenerse de comer el sándwich porque tiene carne cuando él no cree que el hecho de que tenga carne sea una razón para no comerlo?

La explicación, dice Raz, es la siguiente. Ezequiel está afirmando que Verónica no debería comer el sándwich de un modo no comprometido, esto es, desde el punto de vista del vegetarianismo. Así, está afirmando que Verónica debería abstenerse de comer el sándwich, bajo el presupuesto de que el vegetarianismo es verdadero, pero sin comprometerse con que, efectivamente, el vegetarianismo sea verdadero.

Rodríguez-Blanco proporciona, empero, una explicación alternativa del mismo fenómeno familiar. Ezequiel está afirmando que, efectivamente, Verónica debería abstenerse de comer el sándwich. Esto es compatible con que Ezequiel sea omnívoro. Lo que Ezequiel cree es que el hecho de que el sándwich tenga carne es una razón para que los vegetarianos como Verónica no lo coman. El razonamiento sería algo así: i) el hecho de que el sándwich tenga carne es una razón para que los vegetarianos se abstengan 
de comerlo; ii) Verónica es vegetariana; por lo tanto, iii) Verónica debería abstenerse de comer el sándwich.

Sin embargo, hay dos razones para dudar de la explicación alternativa que proporciona Rodríguez-Blanco. En primer lugar, (i) es una razón muy peculiar. ¿Por qué habría de creer que los vegetarianos tienen una razón para no comer carne? Por supuesto, ellos creen que tienen una razón para no comer carne. Pero están equivocados. ¿Es el vegetarianismo auto-validante de modo que quienes creen que tienen razón para no comer carne hacen verdadera su creencia al formarla? Por supuesto, quizás si Verónica sabe que el sándwich tiene carne, puede que tenga una razón para no comerlo, porque lo contrario mostraría debilidad de la voluntad o algo así. Pero, en el ejemplo, Verónica ignora que el sándwich tiene carne; es por eso, precisamente, que Ezequiel le advierte. Por supuesto, Ezequiel cree que tiene una razón para advertirle que el sándwich tiene carne. Quizás quiere evitarle la frustración que sentirá cuando se entere o simplemente quiere ayudarla a vivir de acuerdo a sus ideales. Pero estas son razones para que Ezequiel le advierta a Verónica que el sándwich tiene carne, no son razones para que Verónica se abstenga de comerlo. ${ }^{4}$

En segundo lugar, hay una forma muy simple de testear los méritos de ambas explicaciones y que, a mi juicio, muestra la superioridad de la explicación de Raz. Supongamos que Ezequiel dijera lo siguiente: "No deberías comer ese sándwich porque tiene carne. Aunque, la verdad, está tan delicioso que deberías probarlo". Rodríguez-Blanco debería concluir que Ezequiel está haciendo afirmaciones contradictorias, a saber, que Verónica debe y no debe probar el sándwich. Pero no parece haber nada problemático en lo que dice Ezequiel. Y Raz puede explicar por qué. El primer enunciado es desde el punto de vista del vegetarianismo; asumiendo que el vegetarianismo es verdadero, Verónica no debe comer el sándwich porque tiene carne. El segundo enunciado es comprometido, sin presupuestos. La expresión "la verdad" funciona, precisamente, para marcar que se está abandonando el supuesto que subyacía a la primera oración.

Rodríguez-Blanco argumenta en contra de la interpretación de Raz apelando a un segundo ejemplo:

4 Véase Raz (1999, p. 176). 
Director de una clínica de eutanasia Nazi: Stangl acaba de ser nombrado director de una clínica de eutanasia Nazi. Las prácticas de los Nazis en la clínica le parecen moralmente repugnantes. Pero cree que el derecho Nazi tiene autoridad legítima. Stangl le pide un consejo a su amigo Ezequiel. Ezequiel, a diferencia de Stangl, no cree que el derecho Nazi tenga autoridad legítima. Y, dado el carácter repugnante de las prácticas de los Nazis, cree que Stangl debe renunciar inmediatamente. Sin embargo, le responde que debe seguir siendo director de la clínica (Rodríguez-Blanco, 2021, sección 3).

Rodríguez-Blanco sugiere que, en este caso, a diferencia de lo que sucedía en el caso de la amiga vegetariana, la respuesta de Ezequiel es ininteligible. En el caso de la amiga vegetariana la afirmación de Ezequiel era inteligible, precisamente, porque era plausible sostener que creía que era valioso que los vegetarianos se abstengan de comer carne, aunque los demás no tengamos ninguna razón para hacerlo. Pero aquí una interpretación similar estaría fuera de lugar: no es plausible sostener que Ezequiel cree que los que creen que el derecho Nazi tiene autoridad legítima, tiene la obligación de actuar como requiere, aunque no tenga de hecho autoridad legítima para los demás. Pero Raz, sugiere Rodríguez-Blanco, no puede explicar por qué la respuesta de Ezequiel es problemática. Después de todo, es cierto que, desde el punto de vista del derecho Nazi, Stangl debe permanecer en el cargo. El caso es, en este aspecto, exactamente análogo al de la amiga vegetariana.

Sin embargo, Raz puede perfectamente explicar este sentido de extrañeza. Los enunciados normativos no comprometidos, efectivamente, no sirven para dar consejos, salvo que se los combine con premisas normativas comprometidas. Raz nunca sostuvo lo contrario. Raz nunca sostuvo que Ezequiel, en el ejemplo, estuviera aconsejando a su amiga vegetariana que se abstuviera de comer el sándwich. ¡Por el contrario! Como vimos, Ezequiel podría perfectamente aconsejarle que lo coma de todas maneras. En cambio, Ezequiel está simplemente informándole a Verónica qué debería hacer, bajo el presupuesto de que el vegetarianismo es verdadero. Esto es lo que genera la extrañeza en este caso. Stangl está pidiendo a Ezequiel un consejo. Y, por supuesto, al simplemente decirle lo que debería hacer, asumiendo que el derecho Nazi tiene autoridad legítima, Ezequiel no le está dando ningún consejo. Necesita referirse a lo que efectivamente debe 
hacer Stangl, no simplemente a lo que debe hacer desde tal o cual punto de vista. Y, por supuesto, Ezequiel debería aconsejarle que renuncie a la clínica dadas las aberraciones que allí se cometen.

\section{Rodríguez-Blanco acerca del rol de las normas jurídicas en el razonamiento práctico: algunas observaciones críticas}

El hecho de que las críticas que Rodríguez-Blanco dirige al punto de vista interno hartiano sean inadecuadas no implica que no haya nada que objetar a la teoría de Hart. De hecho, Rodríguez-Blanco tiene razón al sostener que deberíamos examinar el rol que el derecho desempeña en el razonamiento práctico de quienes lo aceptan en mucho mayor detalle que lo que lo hace el propio Hart. También tiene razón al sostener que el no-cognitivismo de Hart es un serio obstáculo para dicho análisis y que la tesis de Hart de que puede aceptarse el derecho por cualquier tipo de razón (e incluso sin razón alguna) es falsa. Pero esto, creo, no tiene nada que ver con la idea de punto de vista interno o con el hecho de que Hart explique las acciones en el contexto jurídico en términos de los estados mentales de los agentes.

Por su parte, el análisis que la propia Rodríguez-Blanco realiza del rol que desempeña el derecho en el razonamiento práctico es, creo, igualmente insatisfactorio. Para Rodríguez-Blanco, muy sintéticamente, los agentes guían su conducta de acuerdo a reglas jurídicas: (i) en virtud de las razones que las fundamentan (i. e. sus grounding reasons), esto es, porque reconocen que se trata de reglas buenas o valiosas; y/o (ii) porque reconocen que es bueno o valioso tener autoridades jurídicas (satisfechas determinadas condiciones), incluso si esta regla en particular no es buena o valiosa (Rodríguez-Blanco, 2014, cap. 8).

Pero ninguna de estos fundamentos explica adecuadamente por qué las reglas jurídicas constituirían razones para la acción. En primer lugar, el hecho de que sea valioso o bueno tener autoridades jurídicas es una razón para contribuir a su formación y preservación, pero no es una buena razón para actuar como requieren. Por lo tanto, (ii) es irrelevante para explicar la fuerza normativa de las reglas dictadas por las autoridades jurídicas. 
En segundo lugar, las razones que justifican o fundamentan una regla no necesariamente constituyen características en virtud de las cuales es bueno o valioso actuar como requiere. Considérese:

Prohibición de la prostitución consentida: Supongamos que pagar por sexo (i.e. por estipulación, prostituir a alguien) está moralmente permitido siempre y cuando la persona a quien se paga para tener sexo (i.e. quien se prostituye) consiente válidamente; pero que, en cambio, cuando la persona que se prostituye no consiente válidamente, prostituirla es moralmente aberrante. Podríamos adoptar una regla jurídica que prohíba solo lo segundo. Pero muchas veces es difícil probar que la persona prostituida efectivamente estaba ejerciendo la prostitución contra su voluntad, por lo que, dado los altos estándares de prueba del derecho penal, la mayoría de las personas que tienen sexo con prostituta/os que están ejerciendo la prostitución contra su voluntad quedarían libres. Una posible solución sería prohibir tanto la prostitución consentida (i. e. prostituir a alguien que consiente válidamente) como la no consentida (i.e. prostituir a alguien que no consiente válidamente), aun reconociendo que, independientemente del derecho, la primera no es moralmente reprochable. Esto reduciría sustancialmente los casos de prostitución no consentida.

Este es un argumento posible para justificar la prohibición de la prostitución consentida. Pero los hechos que justifican prohibirla no son características en virtud de las cuales sea valioso abstenerse de pagar por sexo con el debido consentimiento.

En tercer lugar, aun cuando los hechos que justifican adoptar la regla sean, a su vez, características en virtud de las cuales es valioso actuar conforme a la regla, ello no es suficiente para que la regla sea en sí misma una razón para actuar. Las características en virtud de las cuales es valioso adoptar la regla son también características en virtud de las cuales es valioso actuar conforme a la regla. Pero esas características existen independientemente de la regla: ¿en qué cambia la situación normativa ahora que la regla requiere que realicemos esa acción que era independientemente valiosa?

Por último, hay una suposición común pero errónea que subyace al argumento de Rodríguez-Blanco. Ella sostiene, correctamente, que para que una 
persona guíe su conducta en virtud de reglas jurídicas debe considerar que es bueno o valioso actuar conforme a ellas. Sugiere, además, que ello implica que las reglas son obligatorias, en la medida en que lo son, porque es bueno o valioso actuar como requieren. Esto, empero, no se sigue. Es posible, en efecto, que las reglas sean obligatorias en virtud de otras consideraciones, que no tienen nada que ver con que sea bueno o valioso realizar la acción requerida. Y que sea bueno actuar conforme a las reglas precisamente porque son obligatorias y no que las reglas sean obligatorias porque es bueno actuar conforme a ellas. Esto es, creo, fundamental para entender el modo en que las reglas jurídicas constituyen razones para la acción. Pero el desarrollo de este punto debe esperar, me temo, a otra ocasión.

\section{Bibliografía}

Anscombe, G. E. M. (1963). Intention (2 ${ }^{\mathrm{a}}$ ed.). Cambridge MA: Harvard University Press.

Davidson, D. (2001). Action, Reason, and Causes. En Davidson, D. Essays on Actions and Events ( $2^{\mathrm{a}}$ ed., pp. 12-90). Oxford: Oxford University Press.

Davidson, D. (2001). Freedom to Act. En Davidson, D., Essays on Actions and Events ( $2^{\mathrm{a}}$ ed., pp. 59-74). Oxford: Oxford University Press.

Finnis, J. (1980). Natural Law and Natural Rights. Oxford: Oxford University Press.

Hart, H. L. A. (2012). The Concept of Law (3 $3^{\mathrm{a}}$ ed.). Oxford: Oxford University Press.

Raz, J. (1999). Practical Reason and Norms. Oxford: Oxford University Press. Rodríguez-Blanco, V. (2021). Rastreando las críticas de Finnis al punto de vista interno de Hart: inestabilidad y el "sentido y propósito" de la acción humana en el derecho. Discusiones, 26(1).

Rodríguez-Blanco, V. (2014). Law and Authority Under the Guise of the Good. Oxford: Hart Publishing.

Wittgenstein, L. (1967). Philosophical Investigations. Oxford: Basil Blackwell. 